\title{
El manejo de la urgencia hipertensiva en la central de emergencias no reduce la incidencia de eventos cardiovasculares
}

The management of hypertensive urgency in the emergency department does not reduce the incidence of cardiovascular events

\section{Objetivos}

Describir la proporción de las consultas ambulatorias que fueron por urgencias hipertensivas $(\mathrm{UH})$ y determinar si su manejo en central de emergencias se asocia con mejores resultados, en comparación con el manejo ambulatorio.

\section{Diseño, lugar y pacientes}

Cohorte retrospectiva. Se seleccionaron 58.535 pacientes pertenecientes a un sistema de medicina prepago en EE.UU. que consultaron por UH en el ámbito ambulatorio. Se excluyeron pacientes embarazadas y aquellos que presentaron síntomas al momento de la consulta.

\section{Evaluación de factores pronósticos}

Se definió UH como un registro de presión arterial sistólica y/o diastólica mayor o igual a 180/110 mmHg, sin daño de órgano blanco. La prevalencia de UH se estimó dividiendo las consultas por este problema en el ámbito ambulatorio por el total de consultas du-
Patel KK y col. JAMA Intern Med. 2016;176(7):981-988. rante el período establecido (2008 a 2013). Luego, se dividió a la población en dos grupos: aquellos que luego de la consulta en el ámbito ambulatorio fueron derivados a la central de emergencias (426 pacientes; 0,7\%), y aquellos que fueron enviados a sus hogares (852 pacientes).

\section{Medición de resultados principales}

Se registraron los eventos cardiovasculares mayores (infarto agudo de miocardio y accidente cerebrovascular), la presencia de hipertensión arterial no controlada ( $\geq 140 / 90 \mathrm{mmHg}$ ) y las hospitalizaciones durante los seis meses posteriores a la consulta por UH.

\section{Resultados obtenidos}

El $4,6 \%$ de las consultas ambulatorias fueron por UH. La pérdida de seguimiento fue del $13,6 \%$ y $21,6 \%$ para los pacientes derivados a la central de emergencias o a sus hogares respectivamente. Los resultados principales se resumen en la tabla 1.

Tabla 1. Resultados de interés ajustados* según ámbito de derivación.

\begin{tabular}{|c|c|c|c|c|}
\hline \multirow{2}{*}{\multicolumn{2}{|c|}{ Resultados }} & \multicolumn{2}{|c|}{ Ámbito de derivación } & \multirow[b]{2}{*}{ Valor $\mathrm{P}$} \\
\hline & & Central de emergencias ( $n=426$ ) & Hogar $(n=852)$ & \\
\hline Evento cardiovascular mayor & $\begin{array}{l}7 \text { días } \\
8 \text { a } 30 \text { días } \\
1 \text { a } 6 \text { meses }\end{array}$ & $\begin{array}{l}2 \\
2 \\
4\end{array}$ & $\begin{array}{l}0 \\
0 \\
8\end{array}$ & $\begin{array}{l}0,11 \\
0,11 \\
0,99\end{array}$ \\
\hline Presión arterial no controlada & $\begin{array}{l}1 \text { mes } \\
6 \text { meses }\end{array}$ & $\begin{array}{l}349 \\
213\end{array}$ & $\begin{array}{l}735 \\
393\end{array}$ & $\begin{array}{l}0,04 \\
0,56\end{array}$ \\
\hline Hospitalizaciones & $\begin{array}{l}7 \text { días } \\
8 \text { a } 30 \text { días }\end{array}$ & $\begin{array}{l}35 \\
48\end{array}$ & $\begin{array}{l}40 \\
59\end{array}$ & $\begin{array}{l}0,01 \\
0,01\end{array}$ \\
\hline
\end{tabular}

*Ajustado por edad, sexo, raza, presión arterial sistólica y diastólica, factores de riesgo cardiovascular (hipertensión, diabetes, hiperlipidemia, accidente cerebro vascular o ataque isquémico transitorio, enfermedad renal crónica, diálisis y enfermedad coronaria), y número de antihipertensivos utilizados.

\section{Conclusiones}

La UH es un problema frecuente, pero la tasa de eventos cardiovasculares en ambos grupos fue muy baja (menor al 1\%). Las consultas a la central de emergencias se asociaron con mayor número de hospitalizaciones pero ninguna mejora en los resultados de interés. En ambos grupos la mayoría de los pacientes presentaron hipertensión arterial no controlada a los seis meses. Fuente de financiamiento / conflictos de intereses: no reportados.

\section{Comentario}

Un primer aspecto a remarcar del estudio presentado es la baja tasa de eventos cardiovasculares a corto plazo observada en ambos grupos. Esto refleja la benignidad de lo que comúnmente denominamos "urgencia hipertensiva". En relación al ámbito de manejo, no hubo diferencias significativas entre los dos grupos (hogar vs hospital) con excepción de la mayor tasa de hospitalizaciones del grupo derivado a la central de emergencias. En referencia a esto el Colegio Americano de Emergentólogos sostiene que no es necesario el manejo de la urgencia hipertensiva en central de emergencias y sugiere el seguimiento ambulatorio. La Sociedad Europea de Hipertensión sostiene que un registro aislado elevado de la presión sin evidencia de daño de órgano blanco no debería ser considerado una emergencia y debería tratarse reinstaurando la medicación habitual o intensificando el tratamiento farmacológico habitual'. En el estudio presentado, los pacientes derivados a la central de emergencias se sometieron a 748 estudios complementarios de los cuales sólo el $5,5 \%$ arrojaron resultados anormales. De las 60 tomografías computadas solicitadas, ninguna fue anormal. Esto refleja un gasto in- necesario, exposición a radiación y potenciales daños para nuestros pacientes ${ }^{2}$. Un último dato a remarcar es que a los seis meses de seguimiento más del $60 \%$ de los pacientes presentaron hipertensión arterial no controlada independientemente del grupo al que pertenecían (hospital vs hogar). Esto va en consonancia con los datos que arroja la Organización Panamericana de Salud que muestran que en Argentina, Chile y Brasil solo el $53 \%$ de los pacientes hipertensos está en tratamiento y de ellos, solo el $30 \%$, tiene la presión arterial controlada ${ }^{3}$. La urgencia hipertensiva es un evento que refleja un mal control de la presión arterial y que se asocia a largo plazo con daño de órganos blanco ${ }^{4}$. Sin embargo, ningún estudio ha demostrado que este evento constituya un riesgo en el corto plazo.

\section{Conclusiones de la comentadora}

Desde nuestro rol de médico de cabecera es necesario modificar la mirada sobre la urgencia hipertensiva y abocarnos a mejorar la adherencia y el cuidado crónico de nuestros pacientes hipertensos (ver editorial ad hoc en este número de Evidencia).

María Emilia Espósito [ Senvicio de Medicina Familiar y Comunitaria del Hospital Italiano de Buenos Aires mariaemilia.esposito@hospitalitaliano.org.ar ]

Esposito ME. El manejo de la urgencia hipertensiva en la central de emergencias no reduce la incidencia de eventos cardiovasculares. Evid Act Pract Ambul. 2017;20(4):89. Comentado de: Patel KK, y col. Characteristics and Outcomes of Patients Presenting With Hypertensive Urgency in the Office Setting. JAMA Intern Med. 2016;176(7):981-988. PMID: 27294333.

\section{Referencias}

1. Pak K y col. Acute hypertension: a Systematic review and appraisal of guidelines. The Ochsner Journal 2014;14:655-663.

2. Heath I. Hypertensive Urgency - Is this a useful diagnosis? JAMA Intern Med. 2016;176(7):988-9.

3. Organización Panamericana de la Salud. OPS/OMS pide dar más atención al control de la hipertensión. Washington DC, EE.UU.: Organización Panamericana de la Salud; 14/05/2015. Disponible en URL: http://www.paho.org/uru/index.php?option=com_content\&view=article\&id=950:opsoms-pide-dar-mas-atencion-al-control-de-lahipertension\&ltemid=340 (último acceso octubre 2017).

4. Patel K y col. Characteristics and Outcomes of Patients Presenting With Hypertensive Urgency in the Office Setting. JAMA Internal Medicine JAMA Intern Med. 2016.176(7):981. 